\title{
THE DIAMETER OF ORBITS OF COMPACT GROUPS OF ISOMETRIES; NEWMAN'S THEOREM FOR NONCOMPACT MANIFOLDS
}

\author{
BY \\ DAVID HOFFMAN ${ }^{1}$ )
}

\begin{abstract}
The diameter of orbits of a compact isometry group $G$ of a Riemannian manifold $M$ cannot be uniformly small. If the sectional curvature of $M$ is bounded above by $b^{2}$ ( $b$ real or pure imaginary), then explicit bounds are found for $D(M)$, where $D(M)$ is defined to be the largest number such that: If every orbit $G$ has diameter less than $D(M)$, then $G$ acts trivially on $M$. These bounds depend only on $b$ and the injectivity radius of $M$.

The proofs involve an investigation of various types of convex sets and an estimate for distance contraction of the exponential map on a manifold with bounded curvature.
\end{abstract}

1. Introduction. On a manifold $M$ with metric $d$, consider a compact Lie transformation group $G$. For $y \in M$, let diam $G(y)=\sup \{d(y, g(y)) \mid g \in$ $G\}$ and $D_{G}(M)=\sup \{\operatorname{diam} G(y) \mid y \in M\}$. A classical theorem of Newman states:

"There exists a constant $D(M)>0$, depending only on $M$ and $d$, such that, if $D_{G}(M)<D(M)$, then $G$ acts trivially on $M$."

In other words, the orbits of any nontrivial action cannot be uniformly small with respect to the metric $d$.

If $(M, d)$ is a Riemannian manifold and $G$ is restricted to nontrivial compact subgroups of the isometry group of $M$, it is natural to ask whether $D(M)$ is computable in terms of the Riemannian invariants of $M$. This question has been considered by Mann-Sicks [MS] and $\mathrm{Ku}$ [K]. They were able to bound $D(M)$ from below, provided the action of $G$ was restricted in some manner, or provided $M$ was compact. The main result of this paper can be stated as follows: If the sectional curvature of $M$ is bounded by $b^{2}$, then there exist explicit bounds for $D(M)$ in terms of $b^{2}$ and the injectivity radius of $M$. (Here $b$ may be positive or purely imaginary.) These results are contained in Theorems 2, 3 and 4, and a table at the end of the paper.

Received by the editors November 26, 1974 and, in revised form, March 23, 1976.

AMS (MOS) subject classifications (1970). Primary 57E15.

Key words and phrases. Transformation group, convexity, orbit, Riemannian sectional curvature, Rauch comparison theorem.

(')Partially supported by NSF Grant MCS76-06752. 
We will assume throughout that all our group actions are effective. \$2 develops several notions of convexity, and proves estimates used in later sections. $\$ 3$ deals with fixed point free actions. $\$ 4$ is concerned with orbits near a fixed point on a manifold with bounded curvature. The idea is to compare the orbits of such an action with the orbits of a constructed action on a space form of constant curvature. These new orbits have smaller diameter than the original orbits, and these diameters are computable. This is done in Propositions 3 and 4. $\$ 5$ investigates involutive isometries on complete manifolds.

The author wishes to thank Larry Mann for suggesting this problem to him and for several useful conversations. He also wishes to thank the reviewer for suggesting a simplification of the proof of Theorem 4 and pointing out an oversight in the proof of Lemma 4.

2. Convex sets. The following definitions and notations will be used; for $M$ a Riemannian manifold and $x, y \in M$,

$d(x, y)=$ distance from $x$ to $y$ in $M$,

$S_{\rho}(x)=\{y \mid d(x, y)=\rho\}$,

$B_{\rho}(x)=\{y \mid d(x, y)<\rho\}$,

$K=$ sectional curvature on $M$,

$b=$ real positive or purely imaginary number,

$R(x)=$ "Radius of injectivity of $M$ at $x "=\sup \left\{\rho\left|\exp _{x}\right|_{\|N\|<\rho}\right.$ is injective $\}$,

$$
r(x)= \begin{cases}\min \left(R(x), \pi / 2 \cdot b^{-1}\right), & b>0, \\ R(x), & b \text { pure imaginary, }\end{cases}
$$

$\bar{R}=\sup \{R(x) \mid x \in M\}$. Similarly $\underline{R}, \bar{r}, \underline{r}$.

Given two points $x$ and $y$ in $M$, a geodesic segment $\gamma$ whose length is the distance between $x$ and $y$ will be called a join from $x$ to $y$. We will say " $\gamma$ joins $x$ to $y$ ". For any two points in a complete manifold there is a join. A subset $S$ is called convex if there exists a join between any two points and any join between two points in $S$ lies in $S . S$ is said to be simple if there exists a unique join in $S$ between any pair of points in $S$.

For each $x \in M$, if $d(x, y)<R(x)$ then there exists a unique join from $x$ to $y$; this is an immediate consequence of the definition of $R(x)$. By a classical theorem of Whitehead, each $x \in M$ has a neighborhood which is convex and simple, so (unique) joins exist between points sufficiently close to $x$. Neither of the above results requires completeness. In what follows we will often need to assert the existence of a join between a pair of points. In each case the existence will follow from the assumptions made. If completeness were assumed then between any two points of $M$ there would exist a join. 
However we choose not to make this general assumption and to have our local results applicable to noncomplete manifolds.

Defintion. A pair of points $x, y$ in $M$ is said to satisfy (SLC) if for $\rho=d(x, y)$,

(i) $S_{\rho}(x)$ is a regular hypersurface near $y$, and

(ii) if $\gamma(t)$ is a geodesic tangent to $S_{\rho}(x)$ at $y=\gamma(0)$, the function $\phi(t)=d(x, \gamma(t))$ has a strict local minimum at $t=0$.

Definition. The ball $B_{\rho}(x)$ is strongly locally convex if $\rho<R(x)$ and: for every $0<\tilde{\rho}<\rho$ and $y \in B_{p}(x)$, the pair $x, y$ satisfies (SLC).

REMARK. The condition $\tilde{\rho}<\rho<R(x)$ implies that $S_{p}(x)$ is an embedded hypersurface. The condition (SLC) implies that the second fundamental form $B$ of $S_{\hat{\rho}}(x)$ is positive definite, when defined with respect to the unit outward normal. In other words, $S_{\bar{\beta}}(x)$ is a strictly convex hypersurface.

For $v, w \in T_{q} S_{p}(x), B(v, w)$ may be computed as follows. Let $\delta$ be the unique join from $x$ to $q$. Parametrize $\delta$ with respect to arc length; $\delta(0)=$ $x, \delta(L)=q$. Let $V$ (resp. $W$ ) be the Jacobi field along $\delta$ with $V(0)=0, V(L)$ $=v($ resp. $W(0)=0, W(L)=w)$. Then $B(v, w)=I(V, W)$, where $I(V, W)$ is the index form along $\delta$. If $K<b^{2}$, some positive constant, and

$$
L=\text { length } \delta=d(x, y)<\pi / 2 \cdot b^{-1},
$$

standard comparison methods imply that $I$ is positive definite. If $K<0, I$ is positive definite whatever the length of $\delta$ may be. Specifically, the next lemma is well known.

LEMMA 1. Suppose $K \leqslant b^{2}$ on $M$. Let $x$ and $y$ be such that for $\rho=$ $d(x, y), S_{p}(x)$ is an embedded hypersphere in a neighborhood of $y$ (in particular, there exists $a$ unique join $\delta$ between $x$ and $y$ ). Then if

$$
\rho<\left\{\begin{array}{l}
\pi / 2 \cdot b^{-1}, \quad b>0, \\
\infty, \quad b \text { imaginary, }
\end{array}\right.
$$

the pair $x, y$ satisfies $(S L C)$. If $\rho \leqslant r(x), B_{\rho}(x)$ is strongly locally convex.

For a more detailed account of the above discussion as well as a proof of Lemma 1, see [BC, pp. 247-249].

Mann and Sicks have introduced the following

Definition. A simple convex set $S$ is very strongly convex provided

(1) $\forall x, y, z \in S$, if $\gamma(t)$ joins $x$ to $y$, then the function $\phi(t)=d(z, \gamma(t))$ has no interior maxima.

Proposition 1. Let $S$ be a simple convex set. Suppose that, for every pair of points in $S$, condition ( $S L C)$ is satisfied. Then $S$ is very strongly convex.

Proof. All that needs to be shown is that, under the above hypotheses, $S$ 
satisfies condition (1). Since $S$ is simple and convex, there exists a unique join between any two points $p, q \in S$. Let $\gamma$ be the join of $p$ to $q$, parametrized so that $\gamma(-1)=p, \gamma(+1)=q$. For any $\gamma\left(t_{0}\right),\left|t_{0}\right| \neq 1$, let $\delta$ be the join of $z$ to $\gamma\left(t_{0}\right)$.

(i) If $\delta$ is orthogonal to $\gamma$ at $t_{0}$, then $d(z, \gamma(t))>d\left(z, \gamma\left(t_{0}\right)\right)$ for $\left|t-t_{0}\right|$ small, by (SLC).

(ii) If $\delta$ is not orthogonal to $\gamma$ at $t_{0}$, then $d(z, \gamma(t))>d\left(z, \gamma\left(t_{0}\right)\right)$ for one sign of $t-t_{0},\left|t-t_{0}\right|$ small.

Hence $d(z, \gamma(t))$ has no interior maximum on $[-1,+1]$. This implies (1).

Corollary 1. Suppose $K \leqslant b^{2}$ on $M$.

If $b^{2}>0$ and for all $y \in B_{r(x)}(x), R(y)>2 r(x)$, then $B_{r(x)}(x)$ is very strongly convex. In particular, if $R(y)>\pi \cdot b^{-1}$ for all $y \in B_{r(x)}(x), B_{r(x)}(x)$ is very strongly convex.

If $b^{2}<0$ and $\lambda$ satisfies $2 \lambda<R(y)$ for all $y \in B_{\lambda}(x)$, then $B_{\lambda}(x)$ is very strongly convex.

Proof. Under the curvature assumptions, $K \leqslant b^{2}$, a geodesic of length $<\pi / 2 \cdot b^{-1}$, emanating from a point $x \in M$, must have positive definite index form (no length restriction when $b^{2}<0$ ). This being so, we may use the following lemma of [GKM, p. 160]:

LeMma. Let $B_{\lambda}(x)$ be a normal geodesic ball, centered at $x \in M$, which satisfies: $\forall y \in B_{\lambda}(x), 2 \lambda<R(y)$. Suppose that the index form $I$ is positive definite on all geodesics emanating from $x$ whose length is $\lambda$ or less. Then $B_{\lambda}(x)$ is convex and simple.

The corollary now follows directly from Proposition 1.

It is possible to show very strong convexity of normal balls without the additional assumption of Corollary 1 . The size of these balls is necessarily somewhat smaller.

THEOREM 1. Suppose $K \leqslant b^{2}$ on $M$. Then $B_{r(x) / 2}(x)$ is very strongly convex.

Proof. Recall

$$
r(x)= \begin{cases}\min \left\{R(x), \pi / 2 \cdot b^{-1}\right\}, & b^{2}>0, \\ R(x), & b^{2}<0 .\end{cases}
$$

If $\lambda<r(x) / 2$ the convexity of $B_{\lambda}(x)$ is well known (see [BC, p. 247]). By Lemma $1, B_{\lambda}(x)$ is strongly locally convex. Proposition 1 will allow us to conclude that $B_{r(x) / 2}(x)$ is very strongly convex once we can show that $B_{r(x) / 2}(x)$ is simple. Toward that end, we observe that a pair of points, 
$x, y \in B_{r(x) / 2}(x)$, cannot be conjugate along any join in $B_{r(x) / 2}(x)$ : If $b^{2}<$ $0, M$ is free of conjugate points. If $b^{2}>0$, conjugate points along any geodesic in $M$ must occur at distances of at least $\pi / 2 \cdot b^{-1}$, and any join in $B_{r(x) / 2}(x)$ must have length less than $r(x)$ which is in turn less than $\pi / 2 \cdot b^{-1}$. The simplicity of $B_{r(x) / 2}(x)$ now follows from:

LEMMA 2. Suppose $\bar{B}_{\lambda}(x)$ is an embedded, convex, strongly locally convex ball such that no points of $\bar{B}_{\lambda}(x)$ are conjugate along any join between them. Then $\bar{B}_{\lambda}(x)$ is simple.

Proof. Let $T=\left\{y \in \bar{B}_{\lambda}(x) \mid y\right.$ has a cut point in $\left.\bar{B}_{\lambda}(x)\right\}$ and suppose $T \neq \varnothing$.

Claim. $T$ is a compact set.

Let $T^{1} M$ be the unit tangent bundle for $M$. For $(p, \theta) \in T^{1} M$, let $f(p, \theta)$ be the distance from $p$ to the first cut point of $p$, measured along the geodesic emanating from $p$ with initial direction $\theta$. If $M$ is complete, $f$ is well defined and $f: T^{1} M \rightarrow \mathbf{R} \cup\{\infty\}$ is continuous [Ko, p. 117]. It follows that $F$ : $T^{1} M \rightarrow M \cup\{\infty\}$, given by $F(p, \theta)=\exp _{p} f(p, \theta) \cdot \theta$ is continuous. If $\hat{F}$ is the restriction of $F$ to $\pi^{-1} \overline{B_{\lambda}(x)}$, then $\pi \hat{F}^{-1}\left(\overline{B_{\lambda}(x)}\right)=T$, proving the claim. If $M$ is not complete we may define $f(p, \theta)$ on $\mathscr{D}=\left\{(p, \theta) \in T^{1} M \mid c_{\theta}(t)=\right.$ $\exp _{p} t \theta$ is defined on some interval $I$ which is either equal to $\mathbf{R}^{+}$, or contains the first cut point of $p$ along $\left.c_{\theta}(t)\right\}$. The function $F$ is continuous on $\mathscr{D}$ and since $\overline{B_{\lambda}(x)}$ is an embedded and convex ball, the restrictions $\hat{F}$ of $F$ to that part of $\mathscr{D}$ over $\overline{B_{\lambda}(x)}$ will contain $\overline{B_{\lambda}(x)}$ in its image, so the same proof works.

A more concrete proof, in either case, is outlined as follows: Let $p_{n}$ be a convergent sequence in $T$, converging to $q$. For each $n$, there exist two distinct geodesics $\gamma_{n}$ and $\tilde{\gamma}_{n}$ which join $p$ to $p_{n}$. By appropriate compactness considerations, we may assume that $\gamma_{n}$ converges to a geodesic $\gamma$, and $\tilde{\gamma}_{n}$ converges to a $\bar{\gamma}$, and both $\gamma$ and $\tilde{\gamma}$ join $p$ to $q$. If $\gamma \neq \tilde{\gamma}$, then $q \in T$. If $\gamma=\tilde{\gamma}$ then $q$ must be a conjugate point of $p$ along $\gamma$ and $q$ again must be a cut point of $p$ along $\gamma$.

Since $\bar{B}_{\lambda}(x)$ is embedded, $\alpha=d(x, T)>0$. Since $T$ is compact, there exists a $\tilde{y} \in T$ with $\alpha=d(x, \tilde{y})$. Among all $\tilde{y} \in T$ with $d(x, \tilde{y})=\alpha$, let $y$ be a point such that the distance from $y$ to its cut locus (i.e., $R(y)$ ) is a minimum. Since $\{\tilde{y} \in T \mid d(\tilde{y}, x)=\alpha\}$ is also compact, the existence of such a $y$ is insured. Let $z$ be a cut point of $y$ at minimum distance $\beta=d(y, z)$ from $y$. Then the hypersphere $S_{\beta}(y)$ has a nontransversal self-intersection at $z$ and it follows that there is a geodesic loop, $\gamma$, through $y$ whose midpoint is $z$. Since $\bar{B}_{\lambda}(x)$ is strongly locally convex, no interior point of $\gamma$ can be a point of maximum distance from $x$. Therefore $y$ must be the point of maximum distance from $x$ on $\gamma$ : 


$$
d(z, x)<d(y, x)=\alpha=d(T, x) .
$$

But since $y \in \bar{B}_{\lambda}(x)$ is a cut point of $z, z \in T$, so $d(x, T)<d(z, x)$, a contradiction.

3. Fixed point free actions. The following result is proved in [MS]:

If $G$ is a compact Lie group of isometries of $M$ which acts without fixed points, then no very strongly convex set may contain an orbit of $G$.

By using the results of the previous section (in particular Theorem 1), we may immediately conclude

TheOREM 2. Let $G$ and $M$ be as above with $K \leqslant b^{2}$ on $M$ and $G$ acting without fixed points. Then

(1) For each $x \in M$, diam $G(x) \geqslant r(x) / 2$. Consequently $D_{G}(M) \geqslant \bar{r} / 2$.

(2) Let $b^{2}>0$. The condition $R(y) \geqslant 2 r(x)$, for all $y \in B_{r(x)}(x)$, implies diam $G(x) \geqslant r(x)$. In particular, $R(y) \geqslant \pi \cdot b^{-1}$, for all $y \in B_{r(x)}(x)$, implies $\operatorname{diam} G(x) \geqslant \pi / 2 \cdot b^{-1}$.

(3) Let $b^{2}<0$. If for some $\lambda<R(x), R(y)>2 \lambda$ for all $y \in B_{\lambda}(x)$, then $\operatorname{diam} G(x)>\lambda$.

If $G$ is cyclic, we may prove a stronger result.

TheOREM 3. Let $G$ be a finite cyclic group of isometries of $M$ acting without fixed points. Then for all $x \in M$, diam $G(x) \geq \frac{1}{2} r(x)$. Consequently $D_{G}(M)>$ $\bar{r} / 2$.

The theorem follows from an analogue of the above-mentioned result of Mann-Sicks:

Proposition 2. Let $G$ be a finite cyclic group of isometries. Then any closed, convex, strongly locally convex ball, which contains no fixed points of $G$ contains no orbit of $G$.

If $\rho<r(x) / 2$, Lemma 1 implies that $\bar{B}_{\rho}(x)$ is a closed, strongly locally convex ball. Applying Proposition 2, we may assert that $G(x) \not \subset \bar{B}_{\rho}(x)$. This implies diam $G(x)>\rho$, proving the theorem.

Proof (of Proposition 2). By contradiction. Let $g$ be a periodic isometry generating $G$. Choose $p \in \bar{B}_{\rho}(x)$ such that

$$
d(p, g(p))=\inf \left\{d(y, g(y)) \mid G(y) \subset \bar{B}_{p}(x)\right\} .
$$

Such a $p$ exists by compactness of $\bar{B}_{\rho}(x)$. Also $d(p, g(p))>0$ since $G$ has no fixed points in $\bar{B}_{\rho}(x)$. Let $\gamma$ join $p$ to $g(p)$. The convexity of $\bar{B}_{\rho}(x)$ implies that $\gamma, g \gamma, g^{2} \gamma, \ldots$ all lie in $\bar{B}_{\rho}(x) ; g^{k} \gamma$ joins $g^{k-1}(p)$ to $g^{k}(p)$. We show the closed geodesic loop $\cup g^{k} \gamma$ is actually a smooth closed geodesic. Let $u \in \dot{\gamma}$. Since $G(u) \subset \cup g^{k} \gamma \subset B_{\rho}(x), d(u, g(u))>d(p, g(p))$. On the other hand, 
$d(p, g(p))=d(p, u)+d(u, g(p))=d(g(p), g(u))+d(u, g(p))$. The right-hand side is the length of the broken geodesic segment $\gamma \cup g \gamma$ from $u$ to $g(u)$. Consequently $d(u, g(p))+d(g(p), g(u))>d(u, g(u))$. Since we have already established the reverse inequality, we have $d(u, g(u))=d(u, g(p))+$ $d(g(p), g(u))$. Therefore $\gamma \cup g \gamma$ measures length from $u$ to $g(u)$. From this one concludes that $\gamma \cup g \gamma$ is smooth; if it were not, it could not measure length. The fact that $G=(g)$ consists of isometries implies that $U g^{k} \gamma$ is a smooth (closed) geodesic. Let $p$ be a point on $U g^{k} \gamma$ at maximum distance, $d$, from $x$. It is now clear that strong local convexity is violated, since $U g^{k} \gamma \subset$ $\bar{B}_{p}(x)$, and $\cup g^{k} \gamma$ is tangent to $S_{d}(x)$ at $p$. This concludes the proof of Proposition 2(2).

Under certain additional hypotheses on $M$, one may bound $R$ from below. For example, if either $0<K<b^{2}$, and $M$ is compact, and even dimensional, or $b^{2} / 4<K<b^{2}$ and $M$ is simply connected, then $R>\pi \cdot b^{-1}$ [GKM, p. 227]. Consequently, $r(x)=\pi / 2 \cdot b^{-1}$, and we have

Corollary 2. Suppose either $0<K \leqslant b^{2}$ and $M$ is compact, even dimensional, or $b^{2} / 4<K<b^{2}$ and $M$ is simply connected. Then if $G$ is a compact Lie group of isometries acting without fixed points, every orbit has diameter at least $\pi / 2 \cdot b^{-1}$.

4. Groups with a common fixed point. Our aim is to prove the following

THEOREM 4. Let $G$ be a compact group of isometries acting on $M$ with common fixed point $x$. Suppose $K \leqslant b^{2}$ and $b>0$. Then if $B_{\rho}(x)$ is a convex, embedded ball and if $\rho<\pi / 2 \cdot b^{-1}$, there exists a $y \in M$ satisfying:

$$
d(y, x)=\rho \text { and } \operatorname{diam} G(y) \geq \sqrt{3} b^{-1} \sin b \rho .
$$

Consequently, diam $G \geq \sqrt{3} b^{-1} \cdot \min \{\sin b R(x) / 2, \sqrt{2} / 2\}$.

Remarks. If $K<0$ on $M$, one may use the well-known fact that $\exp _{x}$ stretches distance to estimate orbit size in $M$ by comparison with the orbit size of the induced action of $G$ on $T_{x} M$. This induced action is orthogonal and the orbit size is easy to estimate. The next proposition will allow us to use this idea of proof in case curvature is bounded above by a positive constant.

Proposition 3. Suppose $K<b^{2}$ on a normal coordinate ball $S$ of radius $\rho$, centered at $x \in M$. Further, assume that $\rho=\lambda \cdot b^{-1}$ where $\lambda<\pi / 2$. Let $\tilde{S}$ be a normal coordinate ball of radius $\rho$, centered at a point $y$ in the space form $\tilde{M}$ of constant curvature $b^{2}$. After an isometric identification of $T_{x} M$ with

(2)The author wishes to thank the reviewer for suggesting this method of proof. 
$T_{y} \tilde{M}, e \stackrel{\text { def }}{=} \exp _{x} \circ \widetilde{\exp }_{y}^{-1}: \tilde{S} \rightarrow S$ is a diffeomorphism.

Then $e: \tilde{S} \rightarrow S$ stretches the length of curves, i.e., if $\tilde{\gamma}$ is a curve in $\tilde{S}$, and $\tilde{l}(\tilde{\gamma})$ is the length of $\tilde{\gamma}$ in $\tilde{S}, \tilde{l}(\tilde{\gamma}) \leqslant l(e(\gamma))$.

Furthermore if $S$ is a convex set, e stretches distance, i.e., if $d$ (resp. $\tilde{d})$ is the distance funciton on $M($ resp. $\tilde{M}), \tilde{d}(x, y)<d(e(x), e(y))$.

Proof. Since $K<b^{2}$, no radial geodesic in $S$ (resp. $\tilde{S}$ ) has a conjugate point with respect to $x$ (resp. $y$ ). By the Rauch comparison theorem [see, e.g., [BC, p. 251]], $e$ stretches the length of those tangent vectors on $\tilde{S}$ which are orthogonal to radial geodesics emanating from $y$. It preserves the length of radial tangent vectors. Therefore $e$ must stretch the length of curves. If $S$ is convex, there is a join $\gamma$ between any pair of points $x, y$ in $S$. Hence

$$
\begin{aligned}
d(x, y) & =l(\gamma) \geqslant \tilde{l}\left(e^{-1}(\gamma)\right) \\
& \geqslant \tilde{d}\left(e^{-1}(x), e^{-1}(y)\right) .
\end{aligned}
$$

Proof (OF Theorem 4). Let $x \in M$ be a fixed point of $G$. Choose a point $\tilde{x}$ in $\tilde{M}$, the space-form of constant curvature $b^{2}$. As in Proposition 3, let $\widetilde{\exp }_{\tilde{x}}$ be the exponential map at $\tilde{x}$, and identify $M_{x}$ with $\tilde{M}_{\tilde{x}}$ via a linear isometry. We may then consider $G_{*}=\left\{g_{*}: M_{x} \rightarrow M_{x} \mid g \in G\right\}$, the representation of $G$ on $M_{x}$ via differentials at $x$, as a group of isometries of $\tilde{M}_{\tilde{x}}$. Consider $B_{\pi b^{-1}}(\tilde{x}) \subset \tilde{M}$. Let $\tilde{G}$ denote $G$ acting as a group of isometries of $B_{\pi b^{-1}}(\tilde{x})$ as follows: if $g \in G$ and $\tilde{y} \in B_{z b^{-1}}(\tilde{x})$, let $\tilde{g} \tilde{y}=\widetilde{\exp }_{\tilde{x}} \circ g_{*} \circ \widetilde{\exp }_{\tilde{x}}^{-1} \tilde{y}$.

It is easy to check that $\tilde{G}$ is in fact a group of isometries (i.e. orthogonal transformations) of $B_{z b^{-1}}(\tilde{x})$ which fixes $\tilde{x}$. Therefore, $\tilde{G}$ leaves the hyperspheres $S_{\rho}(\tilde{x})=\{\tilde{y} \in \tilde{M} \mid \tilde{d}(\tilde{x}, \tilde{y})=\rho\}, \rho<\pi b^{-1}$, invariant. Moreover $\tilde{G} \mid S_{\rho}(\tilde{x})$ acts on $S_{\rho}(\tilde{x})$ as a group of orthogonal motions. We claim that there exists a $\tilde{y} \in S_{\rho}(\tilde{x})$ such that diam $\tilde{G}(\tilde{y}) \geqslant \sqrt{3} b^{-1} \sin b \rho$ : First of all, $S_{\rho}(\tilde{x})$ is isometric to a sphere of radius $b^{-1} \cdot \sin b \rho$. Second, we might as well assume that $\tilde{G}$ is cyclic of prime order, $p$. ( $\tilde{G}$ contains such a subgroup and the orbit of a subgroup has diameter at most the diameter of the orbit of $\tilde{G}$.) If $p=2$, then it is clear that $S_{\rho}(\tilde{x})$ contains a point $\tilde{y}$ with diam $\tilde{G}(\tilde{y})=2 b^{-1} \cdot \sin b \rho$. Suppose $p>2$. There must exist a great circle $C \subset S_{\rho}(\tilde{x})$ upon which $\tilde{G}$ acts by rotation through an angle $2 \pi / p$. Let $\tilde{y} \in C$. To estimate the diameter of $\tilde{G}(\tilde{y})$ we need only know the Euclidean distance between two points on a circle of radius $b^{-1} \sin b \rho$, with an angular separation of $(p-1) / p \pi, p>2$. This distance is an increasing function of $p$ and so is minimized when $p=3$. When $p=3$, this distance is equal to $\sqrt{3} b^{-1} \cdot \sin b \rho$. This proves the claim.

The theorem now follows from Proposition 3: Suppose $B_{\rho}(x)$ satisfies the hypotheses of the theorem. In particular $\rho<\pi / 2 \cdot b^{-1}$. Let $\tilde{y} \in S_{\rho}(\tilde{x})$. Then Proposition 3 implies that $\tilde{d}(\tilde{y}, \tilde{g} \tilde{y}) \leqslant d(e \tilde{y}, e \tilde{g} \tilde{y})$, where $e=\exp _{x} \circ \exp _{\tilde{x}}^{-1}$. 
But $e \tilde{g}=\exp _{x} \circ g_{*} \circ \widetilde{\exp }_{\tilde{x}}^{-1}=g \circ \exp _{x} \circ \widetilde{\exp }_{\tilde{x}}^{-1}=g \circ e$. Therefore

$$
d(\tilde{y}, \tilde{g} \tilde{y})<d(e \tilde{y}, g e \tilde{y}),
$$

which implies that $\operatorname{diam} \tilde{G}(\tilde{y})<\operatorname{diam} G(e \tilde{y})$.

5. Group actions which contain an involution. Here we assume that $M$ is complete.

Proposition 4. Suppose $G$ is a group of isometries acting on a complete manifold $M$. Suppose $G$ contains an involution $g$. Then for every $p \in M$, one of the following statements holds

(1) diam $G(p)>R(p)$;

(2) $g$ has a fixed point $x$ with $d(x, p)=\frac{1}{2} d(g(p), p)$ and $\forall \lambda>0, \exists y$ with $d(x, y)=\lambda$ and $\operatorname{diam} G(y)=2 \lambda$;

(3) $g$ has a fixed point $x$ with $d(x, p)=\frac{1}{2} d(g(p), p)$ and there exists a $y$ with $\operatorname{diam} G(y)>2 d(x, y)>R(y)$.

Proof. By completeness of $M$, there always exists a join between any two points in $M$. Moreover, every geodesic is infinitely extendible. Let $g \in G$ with $g \neq 1, g^{2}=1$. Suppose for $p \in M$ there exists more than one join from $p$ to $g(p)$. Then clearly diam $G(p)>R(p)$.

Suppose there exists exactly one join from $p$ to $g(p)$. If $p=g(p)$ then there must be at least one geodesic with $\gamma(0)=p$ such that $g \gamma(t)=\gamma(-t)$ for all $t$. Otherwise $g$ would be the identity map. In this case let $x=p$. If $p \neq g(p)$ let $\gamma$ be the unique join from $p$ to $g(p)$. Since $g \gamma$ joins $g(p)$ to $p$, it must be that $g \gamma=-\gamma$. Consequently the midpoint $x$ of $\gamma$ is fixed. Parametrize $\gamma$ so that $\gamma(0)=x$ and extend $\gamma$ for all time. Thus, whether or not $p$ itself is a fixed point we have a fixed point $x$ of $g$ satisfying $d(x, p)=\frac{1}{2} d(p, g(p))$ and an infinite geodesic $\gamma$ satisfying $\gamma(0)=x$ and $g \gamma=-\gamma$. Either $\gamma$ is a join from $\gamma(t)$ to $\gamma(-t)$ for all $t$, or there exists a least $t_{0}$ for which $t>t_{0}$ implies $\gamma$ does not join $\gamma(t)$ to $\gamma(-t)$. In the former case (2) is satisfied, in the latter case (3) is satisfied.

COROLlaRY 3. If $G$ is a group of isometries acting on a complete manifold and $G$ contains a fixed point free involution, then $D(M) \geq \bar{R}$.

Proof. If $g$ is an involution without fixed points, then (2) and (3) of Proposition 5 are ruled out. Consequently for every $p \in M$, (1) holds. Therefore

$$
\sup \{\operatorname{diam} G(x) \mid x \in M\} \geqslant \bar{R} .
$$




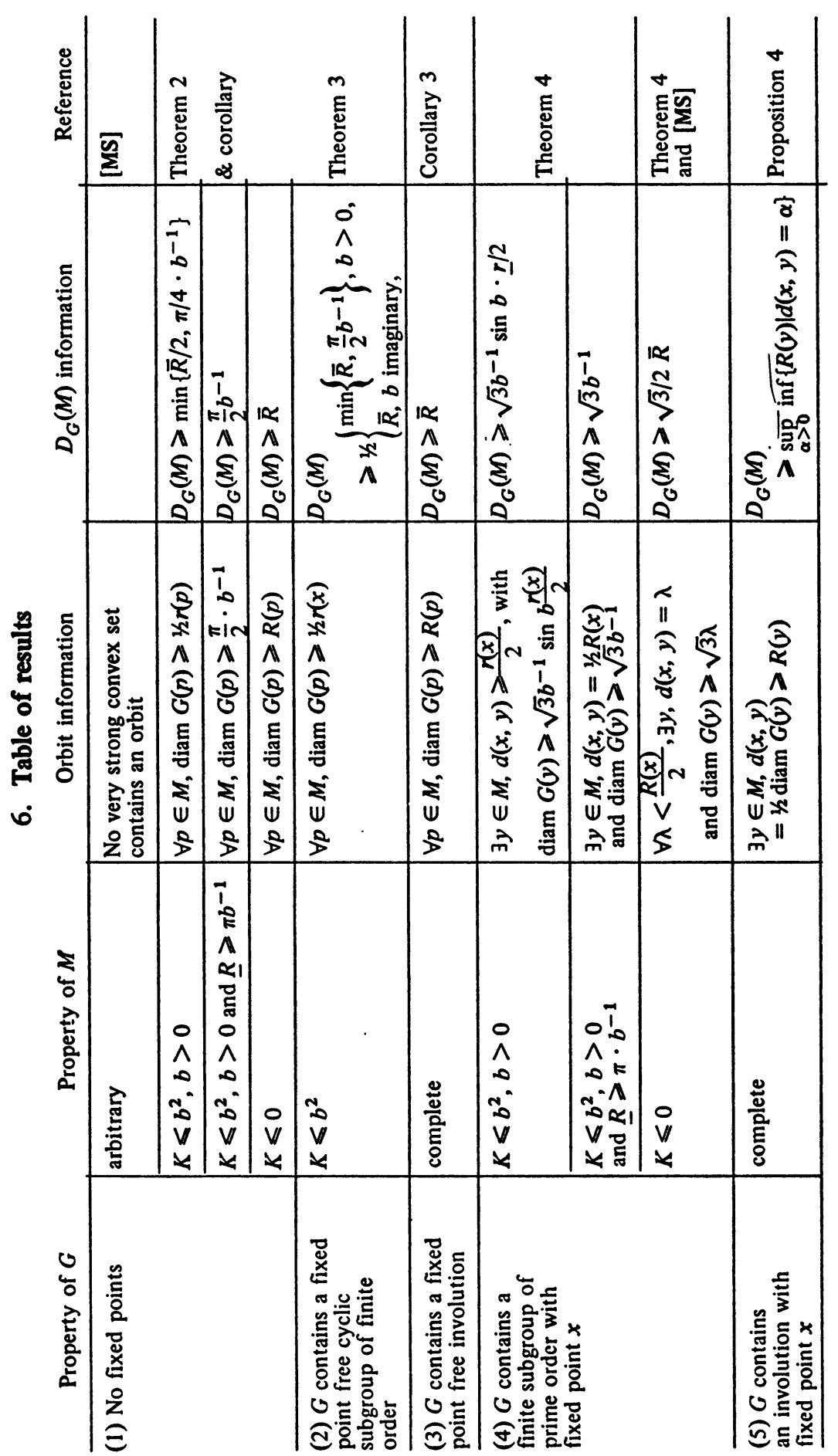


REMARK. If $M$ is a Riemannian manifold with bounded curvature, all possibilities are covered in this table. If $G$ acts without fixed points then (1) applies. If not then it contains a cyclic subgroup of finite order which may or may not act with fixed points. We may use (2) or (4) respectively.

If $M$ is complete with bounded curvature, we can do a bit more. If $G$ acts without fixed points, then (1) applies again. If $G$ itself is finite and of prime order and acts with fixed points, then (4) applies. Otherwise $G$ contains an involution which may, or may not, have fixed points. We may use (3) or (5), respectively.

\section{REFERENCES}

[BC] R. L. Bishop and R. J. Crittendon, Geometry of manifolds, Academic Press, New York, 1964. MR 29 \#6401.

[D] A. Dress. Newman's theorems on transformation groups, Topology 8 (1969), 203-207. MR 38 \#6629.

[GKM] D. Gromoll et al., Riemannsche Geometrie im Grossen, Lecture Notes in Math., vol. 55, Springer-Verlag, Berlin and New York, 1968. MR 37 \#4751.

[K] M. C. Ku, Newman's theorem for compact Riemannian manifolds, Univ. of Massachusetts, Amherst, Mass. (preprint).

[Ko] S. Kobayashi, On conjugate and cut loci, Studies in Global Geometry and Analysis, MAA Studies in Math., vol. 4 (S.S. Chern, editor), Prentice-Hall, Englewood Cliffs, N.J., 1967, pp. 96-112. MR 35 \#3603.

[MS] L. N. Mann and J. L. Sicks, Newman's theorem in the Riemannian category, Trans. Amer. Math. Soc. 210 (1975), 259-266.

Department of Mathematics, University of Massachusetts, Amherst, Massachusetts 01002 